\title{
LV. On the Rev. Mr. Liston's Euharmonic Organ, and his "essay on perfect intonation," just published, for explaining fully the principles of tuning, and of performing upon this organ with perfect harmonies, in almost every possible variety of keys and passages
}

\section{Mr. John Farey Sen.}

To cite this article: Mr. John Farey Sen. (1812) LV. On the Rev. Mr. Liston's Euharmonic Organ, and his "essay on perfect intonation," just published, for explaining fully the principles of tuning, and of performing upon this organ with perfect harmonies, in almost every possible variety of keys and passages, Philosophical Magazine Series 1, 39:169, 373-376, DOI: $10.1080 / 14786441208638146$

To link to this article: http://dx.doi.org/10.1080/14786441208638146

Published online: 27 Jul 2009.

Submit your article to this journal $\sqsubset$

山 Article views: 2 
being only an index to point out the divisions on the circle within the compass : this index being set so as to make the same angle with the needle, as the difference of the true and magnetic meridians, will at all times give the true bearings of any object observed through the two sights $e$ and $f$, which are diametrically opposite, and at the zero of the divided circle; at least whilst the variation of the needle continues the same; but as this alters by the instrument being used in a different tract of country, the brass index $a$ must be turned round a corresponding number of degrees, which are shown by the divided circle in the box. The remaining parts, shown in fig. 2, are $\mathrm{AA}$, the upper ends of the tripod supporting the instrument; $B$ the ball and socket by which the box is adjusted, so as to be horizontal; the stem of the ball has a circle $c$ fixed to it, carrying two sights $g h$, through which the observations are taken; the circle $c$ is divided into degrees, and the compass-box, which turns round upo.a it, has a vernier applying to them : this renders the instrument a theodolite, as any two objects may be observed through the sights $e f$, and $g h$, and the divisions on the circle $c$ will denote the angle between them, and at the samse time the bearing of any object may be taken by observing it through the sights $e f$, and noting the degree pointed out by the brass needle $a$, which is the true bearing required, without any allowance for the variation, as was the case in the common instrument.

I.V. On the Rev. Mr. Liston's Euharmonic Organ, and his "Essay on perfect Intonation," just published, for explaining fully the Principles of Tuning, and of performing upon this Organ with perfect Harmonies, in almost every possible Variety of Keys and Passages. By Mr. John FArey Sen.

\section{To Mr. Tilloch.}

SIR, T He work which has for near a year past, been anxiously expected by great numbers, for explaining the principles and practice of a Musical Scale, without any temperament or imperfections in its harmony, has at length appeared and is to be had of Messrs. Longman and Rees, or any other Bookseller, or at the Music-seliers, and I take the earliest opportunity, of mentioning a few particulars therefrom, in addition to what I communicated in your 
37th volume, page $273 *$, after a perusal of the Rev. Mr. Henry Liston's Mainuscript; with which he favoured me last summer. Since the period alluded to, Mr. William Shield, Mr. Thomas Greatorex, Mr.J. Davy, Mr. Samuel Wesiley, and a great number of other eminent musicians have examined and fully tried the Organ, with 20 pipes in each octave, (through three stops) yielding 60 sounds in each octave, which was exhibited last summer at Messrs. Flight and Robson's, in St. Martin's Lane, and the four professors above mentioned, since voluntarily transmitted to Mr. L. very ample testimonials of the practicability and great value of his improvements on the scale of Keyed Instruments, and which are inserted in his preface.

The interval between the several Minor \& Major consonances $\{6 D \& D, F \& F, b G \& G, 6 B \& B$, and $b C \& c\}$ $\{$ or $2 \mathrm{~d} \& I I, 4$ th \& IV, 5 th $\& V, 7$ th \& VII, \& 8th \&VIII $\}$, Mir. L. denominates a Limma, (page 8) its value in each of the above cases being $T-H$, or $\mathfrak{q}+2 €+2 \Sigma$, or $47 \Sigma$ $+f+4 \mathrm{~m}$, or the Semitone medius $\uparrow$ of $M$. Overend, whose nomenclature I have always followed and wished to recommend others to adopt, for the sake of uniformity and precision in treating on the Scale. The Interval between $\left\{\begin{array}{c}\mathrm{bE} \& \mathrm{E} \text {, and } \mathrm{bA} \& \mathrm{~A} \\ \text { or } 3 \text { rd \& III, and } 6 \mathrm{th} \& \mathrm{VI}\end{array}\right\}$, Mr. L. calls a Grave Limma, (p. 8 ) being $\mathrm{t}-\mathrm{H}$, or $\mathfrak{i}+\mathcal{E}+\Sigma$, or $36 \mathbf{\Sigma}+\mathfrak{f}^{\prime}+$ $3 \mathrm{~m}$, and is the Semitone minor of Overend, \&c.

The Interval between

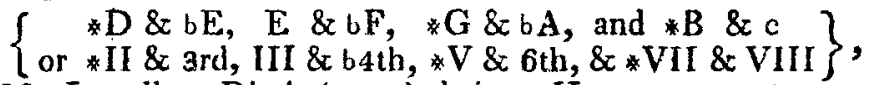
Mr. L. calls a Diesis (p. 10), being $2 \mathrm{H}-\mathrm{t}$, or $2 \mathrm{E}+\Sigma$, or $21 \Sigma+2 m$, and is the Enharmonic Diesis.

The Interval between

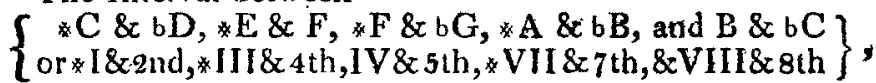
Mr. L. calls a Grave Diesis (p. 10), being $2 \mathrm{H}-\mathrm{T}$, or $\mathrm{E}$ or $10 \Sigma+\mathbf{m}$, and is the Minor Comma.

The Interval between $\left\{\begin{array}{c}b F \quad \& * E, \& b C \& * \mathrm{~B} \\ \text { or b4th \& } \& \text { III, \& 8th \& } * \text { VII }\end{array}\right\}$, being $T+t-3 H$, or $t+\Sigma$, or $26 \Sigma+f+2 m$, is not particularly named by Mr. L. that I observe ; it is the Chromatic Diesis of Dr. Callcott, and its ratio is $\frac{16389}{168} \frac{4}{8}$.

* The specification and description, with a plate, of Mr. L.'s Patent Organ, appeared in our xxxvitth vol. p.328, and vol, xxxviii. p. 47.-EDrTor.

+ Not the Semitone Mitor, or Limma, of my engraved Table of Intervals, vol. xxviii. Plate V. 
and his Essay for explaining its Principles and Use. 375

In like manner as the Minor and Major Intervals of the Scale are at two unequal distances or intervals apart, as mentioned, above, the Sharpen'd Notes of Mr. L.'s Scale are also at two different distances from their naturals*, in different parts of the Scale, and so have flats two different effects, on different notes, respectively: thus,

1 st. The Interval between

$\left\{\begin{array}{l}* \mathrm{C} \& * \mathrm{C}, \mathrm{D} \& * \mathrm{D}, * \mathrm{~F} \& * \mathrm{~F}, \mathrm{G} \& * \mathrm{G}, \text { and B \& } * \mathrm{~B} \\ \text { or } * \text { I \& I, II \&*II,IV \& IV,V \& V, \& VII \& VI }\end{array}\right\}$ is $t-H$, or $\mathfrak{l}+\epsilon+\Sigma$, or $36 \Sigma+f+3 m$, the Semitone minor; and 2nd, between

$\left\{\begin{array}{c}\mathrm{C} \& * \mathrm{C}, \mathrm{E} \& * \mathrm{E} \text {, and } \mathrm{A} \& * \mathrm{~A} \\ \text { or I \& I, III \& } \mathrm{III} \text { \& VI \&*VI }\end{array}\right\}$, is $\mathrm{T}-\mathrm{H}$, or $\mathrm{I}+$ $2 G+2 \Sigma$, or $47 \Sigma+f+4 m$, the Semitone medius, both of which kind of sharps, being on major Intervals, are called Redundant Intervals by Mr. L., and the word major is omitted, as Redundant Second, Redundant Fourth, \& c.

3rd. The Interval between

$\{b D \& b b D+, F \& b F, b G \& b b G+, b B \& b b B, \& C b \& b b C+$ $\{$ ar 2nd \& b2, 4th \& b4, 5th \& $b 5,7$ th $\&$ b7, \& 8 th \& 68$\}$, is $t-H$, the Semitone minor; and $4 t h$, between $\left\{\begin{array}{c}\mathrm{bE} \& \mathrm{bbE}, \& \mathrm{bA} \& \mathrm{~b} \mathrm{bAT} \\ \text { or 3rd \& } \mathrm{b3}, \& 6 \text { th } \& \text { b6 }\end{array}\right\}$, is $\mathrm{T}-\mathrm{H}$, the Semitone medius, both of which kind of flats, being on minor Intervals, are called Diminished Intervals, and the word minor is omitted by Mr. L. in naming such, as Diminished Second, Diminished Fourth, \& $c$.

I must reserve a further account of this interesting volume for another communication, only mentioning, that Messrs. Flights have very nearly fnished a new Organ on $\mathrm{Mr}$. Liston's construction, with $b F$ and other additional Notes, so as considerably to extend the Scale, and perfect more Keys, than on the Organ made in Scotland, which Mr. Liston exhibited last year, and which is still open daily for the inspection and hearing of the lovers of Harmony, who will not neglect, I hope, to treat themselves with examina-

- In all regularly Tempered Scales, there is but one value to Sharps, and the same is also the value of the flats, throughout the scale, and it is called Minor Limma ( 1 ); between these and the next adjoining notes are Major Limmas (L); and every regular Douzeave is composed of $7 \mathrm{~T}+5 \mathrm{l}$ in each octave, in an invariable order.

$\dagger * B, \& c$, or $*$ VII and VIII, $F$ and $F$ or IV and $* I V$, \&ce. do not readily appear to be agreeable to Mr. L.'s principle of naming the Intervals, on which account 1 have stated these intervals as above $C$, although $b b D$, $b \cup G, b b C, b b E$ and $b b A$ are not in his Scale. It is observable, that in double Sharps and double Flats, one of such is $t-H$ and the other $T-H$, so that the value of each $b b$ is the same, viz. the Redundant Limma of Mr. Liston, $=T+t-2 H_{2}=2 f+3 \epsilon+3 \Sigma$, or $83 \Sigma+2 f+7 m$. 
tions of these very curious and Unique Instruments; and hoping, that Mr. L. may receive ample remuneration for his ingenuity and labours, in the Sale of his Work and his Organs.

I remain sir,
Your obedient servant,
12, Upper Crown Street, Westminster,
16th May, 1812.

LVI. Notice respecting the Geological Structure of the Vicinity of Dullin; with an Account of some rare Minerals found in Ireland. By William Fitron, $M$. D. Communicated by L. Horner, Esg. Secretary to the Geological Society.

[Continued from p. 311.]

$T_{\text {He following substances, with the exception of the last }}$ two, have been found within the district to which the preceding observations relate.

1. Vesuvian-(Idocrase, Haüy.) This mineral was observed by Mr.Stephens, in specimens found by me at Kilranelagh in the county of Wicklow ; where it occurs in irregular crystalline masses, in a rock composed of common garnet of a reddish brown colour, of quartz, for the most part greenish, apparently from the admixture of a lamellar fossil of that hue, and a small quantity of yellowish white felspar. The dodecahedral figure of the garnet was very distinct in several of these specimens; but the form of the Vesuvian was not so well exhibited, some indistinct prisms only being observatle; and in general, the crystalline shoots of the latter mineral had assumed a diverging or stelliform arrangement, an appearance which I have not observed in specimens of this substance from other places; but their easy fusibility, lustre, colour, and other characters, were suffrciently decisive of their nature.

I could not discover the original situation of the com. pounc above mentioned at Kilranelagh; but the size, the great weight, and angular form of the blocks consisting of it, render it probable that they were not far removed from their natural place: and the country in that neighbourhood is composed of primitive substances, among which Garnet rock is described by mineralogists, as constituting beds.

It is remarkable, that a compound much resembling that which I have now described, occurs also in the county of Donegal; from whence specimens in the cabinet of the Dublin Society, and that of Dublin College (No. 30), were obtained. 